\section{Acquired bleeding disorders}

Trevor Baglin PhD FRCP FRCPath, Consultant Haematologist, Department of Haematology, Addenbrooke's NHS Trust, Cambridge

Clin Med 2005;5:326-8

The assessment of a patient with a potential acquired bleeding disorder is common in clinical practice. The most common cause is anticoagulant therapy, including antiplatelet drugs. With increasing use of pharmacological thromboprophylaxis in both medical and surgical inpatients and increasing indications for long-term antithrombotic therapy, it is imperative to consider drug-induced bleeding during the initial evaluation of abnormal bleeding. Some patients who bleed abnormally during or after surgery have a mild underlying heritable haemostatic defect; an important aspect of assessment is therefore to determine whether there is a heritable defect with late clinical onset. Most congenital disorders of haemostasis are mild (eg von Willebrand disease) and abnormal bleeding may not become manifest until there is a haemostatic challenge such as surgery or menstruation. Effective treatment depends on a critical assessment of the extent and nature of bleeding.

Presentation of an acquired bleeding disorder varies between acute unexpected bleeding during or immediately after surgery ${ }^{1}$ to unusual or excessive bruising, purpura, epistaxis or gum bleeding developing over several months. In all cases a comprehensive history is needed to assess the nature and extent of the bleeding, to guide the clinical examination and determine the most appropriate use of investigations. The major issues to be determined are:

- Is haemostatic capacity reduced or is there a non-haematological cause for bleeding?

- If haemostatic capacity is reduced, is it due to a heritable defect with late clinical onset or the result of a newly acquired defect?
- If newly acquired, is it due to an anticoagulant drug?

- If not due to reduced haemostatic capacity, what are the likely circumstances that led to abnormal bleeding?

\section{Assessing haemostatic capacity}

The history is of primary importance in determining if haemostatic capacity is reduced. Standard laboratory tests such as prothrombin time (PT) and activated partial thromboplastin time (APTT) are insensitive to mild but clinically significant reductions in haemostatic capacity, and measurement of platelet count gives no indication of platelet function. These tests are not influenced by levels of von Willebrand factor. Therefore, it is on the basis of the history that a clinician must decide the extent of laboratory testing required. The haemostatic response to previous challenges such as surgery, dental extraction and menstruation should be determined from the history. The family history is also important as it may indicate a likely underlying heritable defect. A complete drug history should be taken. The bruising history is difficult to quantify and not particularly informative. Similarly, a history of epistaxis as a child has a low predictive value for an underlying bleeding disorder. Despite their insensitivity, the PT, APTT and platelet count should be measured; if they are normal but the history is highly suggestive of an underlying bleeding disorder, a haematologist should be consulted regarding a more comprehensive laboratory assessment of haemostatic capacity.

It is essential to identify any nonhaematological cause for bleeding, such as a surgical bleeding point in a patient with acute bleeding, a structural vessel defect as in hereditary haemorrhagic telangiectasia or a major medical illness such as liver or renal impairment.

\section{Acquired or heritable defects}

Some patients who bleed abnormally during or after surgery have a mild underlying heritable haemostatic defect. The most common heritable bleeding tendency is a low von Willebrand protein level. This may be due to genetic mutation of the von Willebrand factor gene, or more commonly is the effect of other genetic factors such as blood group O.,3

Whatever the reason, the level of von Willebrand protein appears to be an important continuous variable influencing the clotting phenotype. The first apparent manifestation of this may be excessive surgical bleeding, as a result of which the patient is considered to have an acquired bleeding disorder. The history may be informative, such as a detailed menstrual history in women. It can be difficult to establish a diagnosis of a mild reduction in von Willebrand protein in the immediate postoperative period as levels rise as part of the stress response. Consequently, it is prudent to re-evaluate patients several weeks after an episode of abnormal surgical bleeding.

\section{Drug-induced bleeding}

The most common cause of an acquired bleeding disorder is anticoagulant therapy, eg warfarin. Platelets are integral to thrombin generation; antiplatelet drugs can be considered as anticoagulants, hence their ability to prevent thrombosis. Bleeding risk is in part determined by the potency of antiplatelet activity: for example, the bleeding risk associated with a fibrinogen receptor antagonist (IIb/IIIa inhibitor) is far greater than with aspirin or an adenosine diphosphate receptor antagonist such as clopidogrel. Individual response to antiplatelet therapy is extremely variable and even aspirin or clopidogrel will produce a significant bleeding tendency in some patients.

Approximately one in 100 of the UK population now receives long-term oral anticoagulant therapy. Overanticoagulation, often due to intercurrent illness and antibiotic use, ${ }^{4}$ is probably responsible for the majority of lifethreatening bleeds due to antithrombotic therapy.

\section{Surgical bleeding}

Postoperative bleeding is a common clinical problem. It is essential to examine 
the drug and infusion charts and check that the dose of any drug that may affect haemostasis is correct. It is also imperative to determine if the site of surgery is the only site of bleeding. If this is the case (eg no bleeding from venepuncture sites or an endotracheal tube) and there is no history of previous abnormal bleeding, it is important to keep the possibility of anatomical surgical bleeding as a likely possibility depending on the results of coagulation tests. In some cases of severe bleeding the patient may have to return to theatre to look for a bleeding point.

\section{Critically ill patients}

There are many potential acquired disorders of haemostasis in critically ill patients. For example, a coagulopathy due to vitamin $\mathrm{K}$ deficiency occurs within a few days in those receiving no oral intake. ${ }^{5,6}$ Parenteral vitamin K supplementation should be used routinely to prevent bleeding. Many critically ill patients develop disseminated intravascular coagulation (DIC).

\section{Disseminated intravascular coagulation}

The major manifestations of DIC are end-organ damage due to microvascular thrombosis, but the most readily apparent clinical manifestation is often bleeding due to the consumptive coagulopathy. DIC is a clinical diagnosis supported by the results of laboratory investigations. ${ }^{7}$ The most important aspect of treatment is that of the underlying cause (eg sepsis), although fresh frozen plasma (FFP) and platelet concentrates are used to treat bleeding or prevent haemorrhage associated with planned invasive procedures.

\section{Massive transfusion}

A dilutional coagulopathy resulting in deficiency of clotting factors and platelets will cause abnormal bleeding in patients receiving large amounts of plasma expanders and red blood cells even in the absence of DIC. ${ }^{8}$ It is important to give replacement therapy with FFP and platelet concentrates, guided by repeated measurement of the PT, APTT and platelet count.

\section{Thrombocytopenia}

Many drugs result in a reversible idiosyncratic thrombocytopenia, in most cases mild and which do not cause bleeding. Notable exceptions are quinine- and gold-induced thrombocytopenia which are severe. ${ }^{9}$ An evaluation of drug history and cessation of possibly implicated drugs are both essential in patients with acquired bleeding found to be thrombocytopenic. Cytotoxic drugs produce a dose-dependent suppression of bone marrow platelet production and thrombocytopenic bleeding is common in oncology practice. Bone marrow suppression and bone marrow failure syndromes (eg aplastic anaemia and myelodysplasia) often result in production of dysfunctional platelets; the bleeding tendency in these conditions is significantly greater than in patients with thrombocytopenia and an uncompromised marrow, such as occurs in immune thrombocytopenic purpura (ITP) (see below).

\section{Acquired inhibitors}

Acquired inhibitors are rare and most often autoantibodies. Platelet autoantibodies result in shortened platelet survival and ITP. 2,10 The bleeding manifestations of ITP are variable and often mild compared with thrombocytopenia associated with inadequate platelet production (see above). Rarely, an autoantibody to a specific clotting factor, such as factor VIII, produces a severe acquired bleeding disorder.

\section{Treatment of acquired bleeding disorders}

\section{Acute bleeding}

Effective treatment depends on a critical assessment of the extent and nature of bleeding, if not a diagnosis. When a specific haemostatic abnormality is identified, appropriate therapy can be given. Drugs that cause bleeding should be stopped and non-haematological causes of bleeding managed appropriately, for example by dialysis and elevation of

\section{Key Points}

Acquired bleeding disorders are
common in clinical practice
Standard laboratory tests such as
prothrombin time and activated
partial thromboplastin time are
relatively insensitive to clinically
significant reductions in
haemostatic capacity
Anticoagulant use is the most common
cause of drug-induced bleeding
Efforts should be made to identify the
presence of specific haemosta......................................................................................................................................
abnormalities, with correction of
these using appropriate treatment
In critically ill patients abnormal
bleeding is often multifactorial,
including vitamin K deficiency and
disseminated intravascular
coagulation

KEY WORDS: anticoagulant, bleeding, haemophilia, platelets, surgery

haematocrit in patients with renal failure. Vitamin $\mathrm{K}$ should be given to critically ill patients and patients with liver disease. Supportive care, with judicious use of FFP and platelets, should be given to patients with severe coagulopathy, such as DIC, whilst the underlying condition is being treated. Patients with overt haematological disorders such as myelodysplasia, ITP or factor VIII inhibitors need to be managed by, or in conjunction with, a haematologist. Pharmacological agents can be used to increase haemostatic capacity, but only by clinicians with appropriate experience. Such drugs include desmopressin (DDAVP), ${ }^{11}$ tranexamic acid and aprotinin. Experimental approaches such as recombinant factor VIIa may also be tried. $^{12}$

\section{Non-acute bleeding}

It is important both to identify the circumstances that contribute to abnormal bleeding and to determine the likelihood of an underlying persistent bleeding tendency as this will influence future management, for example at times of surgery or decisions regarding antithrombotic therapy. A comprehensive drug history 
should identify drugs that may have to be stopped. Some individuals are particularly sensitive to the usually mild anticoagulant effect of aspirin. A single episode of abnormal surgical bleeding may not be readily explained, but this should be taken into consideration at times of future surgery so that mechanical rather than pharmacological thromboprophylaxis is used and any antiplatelet therapy stopped.

\section{References}

1 Koh MB, Hunt BJ. The management of perioperative bleeding. Review. Blood Rev 2003;17:179-85.

2 Provan D, Singer C, Baglin T, Lilleyman J. Oxford Handbook of Clinical Haematology. Oxford: Oxford University Press, 2004.

3 Sadler JE. von Willebrand disease type 1: a diagnosis in search of a disease. Review. Blood 2003;101:2089-93.

4 Baglin T. Avoiding overanticoagulation: knowing your antibiotics. Thromb Haemost 2002;88:703-4.

5 Alperin JB. Coagulopathy caused by vitamin $\mathrm{K}$ deficiency in critically ill, hospitalized patients. JAMA 1987;258:1916-9.

6 O'Shaughnessy D, Allen C, Woodcock T, Pearce $\mathrm{K}$ et al. Echis time, under-carboxylated prothrombin and vitamin $\mathrm{K}$ status in intensive care patients. Clin Lab Haematol 2003;25:397-404.

7 Levi M, Ten Cate H. Disseminated intravascular coagulation. Review. $N$ Engl J Med 1999;341:586-92.

8 Hirshberg A, Dugas M, Banez EI, Scott BG et al. Minimizing dilutional coagulopathy in exsanguinating hemorrhage: a computer simulation. J Trauma 2003;54:454-63.

9 Garner SF, Campbell K, Metcalfe P, Keidan J et al. Glycoprotein V: the predominant target antigen in gold-induced autoimmune thrombocytopenia. Blood 2002;100:344-6.

10 British Committee for Standards in Haematology, General Haematology Task Force. Guidelines for the investigation and management of idiopathic thrombocytopenic purpura in adults, children and in pregnancy. Br J Haematol 2003;120:574-96.

11 Mannucci PM. Desmopressin (DDAVP) in the treatment of bleeding disorders: the first 20 years. Review. Blood 1997;90:2515-21.

12 Ghorashian S, Hunt BJ. 'Off-license' use of recombinant activated factor VII. Review. Blood Rev 2004;18:245-59.

\section{Myeloproliferative}

\section{disorders}

Jim Murray FRCP FRCPath,

Consultant Haematologist, University of Birmingham NHS Foundation Trust

Clin Med 2005;5:328-32

Myeloproliferative diseases are clonal stem cell disorders characterised by proliferation of one or more of the erythroid, granulocytic or megakaryocytic cell lines resulting in increased numbers of red blood cells, granulocytes or platelets in the peripheral blood. Extramedullary haemopoiesis may also occur in the liver and spleen. The major non-leukaemic myeloproliferative disorders are polycythaemia vera (PV), essential thrombocythaemia (ET) and myelofibrosis. ${ }^{1}$ These conditions overlap, with the potential for further clonal evolution to either myelofibrosis or acute myeloid leukaemia (AML) (Fig 1). ${ }^{2}$

\section{Polycythaemia}

The term polycythaemia refers to a number of conditions where the haemoglobin and haematocrit are excessively raised. Patients with a persistently raised haematocrit will usually require a red cell mass estimation to confirm whether there is a genuine or absolute increase in red cell volume. A diagnosis of absolute erythrocytosis can be assumed, however, if the haematocrit exceeds 0.60 in men or 0.56 in women.

The classification of polycythaemia has proved confusing in the past, with the use of terms such as secondary, relative or apparent polycythaemia. The term 'relative polycythaemia' or erythrocytosis should be reserved for states of dehydration, and 'apparent polycythaemia' where there is a raised haematocrit but the red cell mass is within the normal range. The causes and classification of absolute polycythaemia are shown in Table 1.

\section{Clinical features}

The median age of presentation is 55-60 years, with an equal sex incidence. Vascular occlusive events are a common presenting feature but there may also be haemorrhage and, in about two-thirds of patients, an enlarged spleen. A useful clinical pointer is the presence of aquagenic pruritus when patients complain of intense itching after a hot bath or shower. Increased uric acid production is not uncommon. The natural history includes progression to myelofibrosis in about $30 \%$ of individuals and the development of AML in about $10 \%$.

\section{Key Points}

The non-leukaemic myeloproliferative disorders are clonal stem cell disorders and have considerable overlap

All these disorders can undergo further clonal evolution to develop either myelofibrosis or acute myeloid leukaemia

Venesection is the standard initial therapy of patients with a raised haematocrit

Essential thrombocythaemia remains a diagnosis of exclusion in the absence of a single confirmatory test

The role of hydroxyurea in increasing the risk of acute leukaemia is still far from clear

Further randomised controlled trials are required to define the indications for and the timing of treatment

KEY WORDS: clonal evolution, extramedullary, haematocrit, leuco-erythroblastic, leukaemogenesis, non-leukaemic, transplantation 